\title{
Education, occupational class and cognitive decline in preclinical dementia
}

\author{
Dorina Cadar ${ }^{1}$, Andrea M. Piccinin ${ }^{2}$, Scott M. Hofer ${ }^{2}$, Boo Johansson ${ }^{3}$ and Graciela \\ Muniz-Terrera $^{1}$ \\ ${ }^{1}$ MRC Unit for Lifelong Health and Ageing at University College London, UK \\ ${ }^{2}$ Department of Psychology, University of Victoria, Victoria, Canada \\ ${ }^{3}$ Department of Psychology, University of Gothenburg, Gothenburg, Sweden
}

*Requests for reprints should be addressed to Dr Dorina Cadar MRC Unit for Lifelong Health and Ageing, London, 33 Bedford Place, LondonWC1B 5JU, UK, (e-mail: d.cadar@ucl.ac.uk).

\section{Funding}

This work was supported by the following funding agencies: Alzheimer's Society [Grant number 144], the Medical Research Council [Unit Programme number MC_UU_12019/1], the US National Institutes of Health National Institute of Aging [P01AG043362], the Swedish Research Council for Health, Working Life and Welfare and by the National Institute on Aging of the National Institutes of Health under award number P01AG043362 for the Integrative Analysis of Longitudinal Studies of Aging (IALSA) research network. The content is solely the responsibility of the authors and does not necessarily represent the official views of the National Institutes of Health or other funding bodies mentioned above. 


\begin{abstract}
We investigated education and occupational influences, as markers of cognitive reserve, in relation to cognitive performance and decline on multiple fluid and crystallized abilities in preclinical dementia. From the total sample of 702 participants, from OCTO-Twin, Sweden, aged 80+ at baseline in 1992-1993; only those who developed dementia during the study period $(\mathrm{N}=127)$ were included in these analyses. Random effects models were used to examine the level of performance at the time of dementia diagnosis and the rates of decline prior to diagnosis. Results demonstrated that both fluid and crystallized abilities decline in preclinical stages and education and occupational class have independent moderating roles on the cognitive performance at the time of diagnosis, but not on the rates of decline.
\end{abstract}

Keywords: dementia, aging, preclinical, cognitive decline, fluid and crystallized abilities 


\section{Introduction}

Although most individuals are likely to experience decline in their cognitive abilities as they age, greater cognitive impairment highlights the first signs of neuropathological damage and the potential progression to neurodegenerative conditions such as dementia or Alzheimer's disease.

Studies comparing the risk of developing late onset Alzheimer's disease in identical twins compared with non-identical twins show a heritability of around 0.6, so the genetic component of late onset Alzheimer's disease is around 60 per cent with the other 40 per cent coming from environmental factors (Bergem, Engedal, \& Kringlen, 1997). The concordance of Alzheimer's disease (AD) in monozygotic (MZ) twins is estimated to be $80 \%$, suggesting high heritability patterns. However, the genetic patterns seem to diverge as MZ twins become older, so the significant heritability remains unexplained. In this context, environmental influences such as education or lifestyle behaviors have been proposed to have a long term influence on genetic alterations (Bjornsson, Fallin, \& Feinberg, 2004; Sweatt, 2010).

A proposed explanation is that cognitive reserve may moderate the effects of neocortical and limbic neuropathology (Stern, 2009). According to this hypothesis, complex mental activities developed across the lifespan (through educational attainment, occupation or participation in leisure activities), could allow compensatory cognitive mechanisms to be employed, when confronted with underlying neurodegenerative dysfunction (Stern, 2002). Education and socioeconomic position are often considered proxies for cognitive reserve since they are key markers for the environmental experiences that have an effect on cognition (Albert, Jones, Savage, Berkman, Seeman, Blazer et al., 1995), cognitive decline (Clouston, Kuh, Herd, Elliott, Richards, \& Hofer, 2012; Hall, Derby, LeValley, Katz, Verghese, \& Lipton, 2007; Richards \& Deary, 2005; Wilson, Li, Aggarwal, Barnes, McCann, Gilley et al., 2004) and dementia incidence (Tervo, Kivipelto, Hanninen, Vanhanen, Hallikainen, Mannermaa et al., 2004). Cognitive reserve theory has been supported by the results of a systematic review, which emphasized that complex patterns of mental activity across life stages are associated with a significant reduction in dementia incidence and less severe clinical or 
cognitive changes in the presence of age-related neurodegeneration (Valenzuela \& Sachdev, 2006). In addition, several prospective longitudinal studies have shown that a number of individuals with no evidence of symptomatic cognitive impairment who meet clinicopathologic criteria for Alzheimer's disease can be identified (Mortimer, 1997).

Although, the existing evidence regarding a protection against faster cognitive decline in healthy individuals is well documented, the evidence related to preclinical dementia remains unclear. It is not inconceivable, that the difference in rate of neuropathological advancement balances the difference in the association between cognitive ability and neuropathology, which could lead to similar slopes in cognitive decline. According to the cognitive reserve hypothesis, a person's cognitive test score depends on both premorbid ability level and the amount of neuropathology accumulated, which increases with age. However, after a certain inflection point or accumulation stage, cognitive ability starts to decline and this decline could start later and proceed more quickly for those with high cognitive reserve (Stern, 2012). This assumes that the rate of neuropathology progression is constant regardless of level of cognitive reserve. When the rate of neuropathological decline is slower in those with higher cognitive reserve, due to compensatory mechanisms (Valenzuela \& Sachdev, 2006), the effective rate could be slower with more cognitive reserve. The discrepancy in findings could also be related to the methodology employed or to the type of neuropsychological tests examined (Hofer \& Piccinin, 2010; Piccinin, Muniz, Matthews, \& Johansson, 2011).

Regarding the latter, the aging process has a differential effect on cognitive functioning in later life according to the different type of cognitive abilities (fluid or crystallized). In general, fluid abilities such as processing speed, memory, visuospatial ability and attention are considered to be age sensitive, while crystallized knowledge such as verbal abilities (to understand written and spoken language), are less age sensitive and tend to remain stable into old age (Cattell, 1963; Crawford, Deary, Starr, \& Whalley, 2001; Horn \& Cattell, 1967). Consequently, these mental abilities tend to present different slopes of decline in relation to age (Anstey \& Low, 2004). Several 
epidemiological studies showed that an accelerated decline was mostly observed with the fluid cognitive abilities, and therefore a steeper decline in these functions was associated with an increased risk of developing dementia (Fleisher, Sowell, Taylor, Gamst, Petersen, \& Thal, 2007; Petersen, 2004; Roberts, Karlawish, Uhlmann, Petersen, \& Green, 2010). However, less is known about the rate of decline between fluid and crystallized functions prior to dementia diagnosis, when the process of neurodegeneration is considered to vary substantially from normal aging.

In this context, it is essential that population samples are well-defined and that cognitive trajectories are investigated within samples of subpopulations (e.g., healthy individuals or dementia cases). Process-based approaches to analyze longitudinal data represent unique opportunities to evaluate theories of aging, such as by testing key predictions about the rates of cognitive decline in the period prior to dementia diagnosis and how cognitive performance (one variable) changes in relation to proximity to dementia (another variable) (Hofer \& Piccinin, 2010; Sliwinski \& Mogle, 2008). Modeling cognitive change in relation to the time prior to clinical diagnosis could provide a more informative-descriptive and explanatory modeling process, compared to the age-based models. It offers an improved indication of the rates of cognitive decline longitudinally, as there is an important non-age-graded process driving the cognitive change in the years prior to dementia diagnosis, reflecting the progression of the disease in older individuals.

The aim of this study was to evaluate the associations of different cognitive reserve variables (e.g. education, occupational class) with fluid and crystallized abilities in the period prior to dementia. With this purpose, we considered a process-based approach (Sliwinski \& Mogle, 2008), in which we modeled the decline in all neuropsychological tests available in the Octogenarian Twins Study (also known as the OCTO-Twin), using random effects mixed models, where cognitive scores were aligned according to time to dementia diagnosis from study entry.

We hypothesized that both higher education (more years) and higher occupational levels (intermediate or professional levels) may protect against the adverse effects of accumulating neuropathology and could moderate the rate of cognitive decline measured in both fluid and 
crystallized abilities in the preclinical stages of dementia. We also hypothesized that those with higher cognitive reserve will have better performance on both fluid and crystallized measures at the time of diagnosis.

\section{Method}

\section{Study population}

Participants were drawn from the longitudinal Origins of Variance in the Old-Old: Octogenarian Twins (OCTO-Twin Study), based on the oldest cohort of the Swedish Twin Registry. The full sample includes 702 participants, with 351 complete twin pairs born in 1913 and earlier, who became 80 years of age during the first wave of data collection (1991-1993). Participants have been re-assessed every two years across the study period (1991-2001) on up to five occasions. The average rate of attrition from one testing wave to the next was $20 \%$ (10\% per year), primarily due to death. Full details of the study population characteristics have been published previously (McClearn, Johansson, Berg, Pedersen, Ahern, Petrill et al., 1997; Pedersen, Lichtenstein, \& Svedberg, 2002).

We analyzed data from the subsamples of individuals who developed dementia during the course of the study and who were free of dementia at study entry. From the total sample of OCTOTwin Study, 225 individuals had dementia, representing 32\% of the total sample. From these, 98 people had already been diagnosed with dementia at the study entry and were excluded from the main analyses. The remaining127 participants who developed dementia during the study period represent the subpopulation sample used for these analyses.

\section{Procedure}

Ethical approval was received from the Ethics committee at Karolinska Institute in Stockholm and the Swedish Data Inspection Authority in Sweden. All participants signed informed consent forms (Johansson \& Zarit, 1995).

Dementia diagnosis was established by clinicians according to the revised third edition of the Diagnostic and Statistical Manual of Mental Disorders (DSM-III). During the interviews across 
the study period, participants were asked for permission to review their medical records, ordered from hospitals, outpatient clinics, district physicians and primary health care. A multi-disciplinary team consisting of a physician and two neuropsychologists reviewed testing results and medical records, including medicine use and self-reported information about diseases at each new wave (van den Kommer, Bontempo, Comijs, Hofer, Dik, Piccinin et al., 2009).

\section{Study variables}

The OCTO-Twin Study encompasses a broad spectrum of cognitive and behavioral measures, assessed at each of the five waves during the study period.

Neuropsychological Measurements consisted of multiple tests of fluid abilities and crystallized abilities, as well as the Mini-Mental State Examination (MMSE) (Folstein, Folstein, \& McHugh, 1975). Fluid abilities including visuospatial ability inductive reasoning and memory were measured by Block Design, Figure Logic, Swedish Clock, Digit-Symbol, Prose Recall, Memory Recall, Memory Recognition and Memory Correspondence. Crystallized abilities such as knowledge ability and verbal meaning were measured by Information and Synonym tests. Participants were tested in their home by medical research nurses who were specially trained. A summary of these cognitive tests is provided as online material.

Education was measured as the number of years the individual went to school.

Occupational class was measured during the home interview. The question asked was: "What has been your main occupation for most of your working life (during the longest period)?" and the responses were coded into low (manual occupations), medium (non-manual occupations) and high (intermediate and professional occupations).

\section{Data analyses and analytic approach}

We evaluated the whole range of neuropsychological tests. Random effects mixed models were fitted to each cognitive outcome as a function of time to dementia diagnosis, which was calculated for each wave of data collection in relation to the time of dementia diagnosis, which was set at zero to represent the intercept. Between-person differences in age and in time to dementia 
diagnosis were separated from within person changes by adjusting the level and rate of change by age at study entry and by time to dementia diagnosis from study entry (Piccinin et al., 2011).

We examined education (years of formal education) and occupational class as markers of cognitive reserve, and a number of common demographic covariates including sex, age at study entry, and time to dementia diagnosis from study entry. Male and low occupational histories were used as reference categories. Age and time to dementia diagnosis from study entry were mean centred. Given this model specification, the intercept represents performance at the time of dementia diagnosis for an individual with values of zero on all covariates (i.e., for an 83 years old man of low occupational class and 7 years of education who entered the study 5 years before dementia diagnosis) and the linear slopes represent annual rate of change (e.g., increase, decrease) per year closer to the time of dementia diagnosis. All analyses were performed using Stata software, Version 13 (StataCorp, 2013).

Table 1 about here

\section{Results}

Table 1 provides a summary of participant characteristics. Information is provided for the full sample as well as for the dementia-free, demented at study entry and incident case groups for comparative purposes. Only $10 \%$ of those who had dementia at study entry and $12 \%$ of incident cases during the study period had a higher occupational class. Occupation and dementia incidence were found to be independent $\left(\mathrm{X}_{(2)}^{2}=8.01, \mathrm{p}=0.09\right)$. The relation between sex and dementia incidence was significant (Chi-square with two degrees of freedom $X_{(2)}^{2}=8.96, p \leq 0.01$ ) suggesting that women were more likely to have already been diagnosed with dementia at baseline.

There were significant differences in years of education and baseline age by dementia status $($ ANOVA, $\mathrm{F}(2,655)=5.58, \mathrm{p}=0.003$ and $\mathrm{F}(2,701)=7.38, \mathrm{p} \leq 0.001$ respectively $)$. Post hoc analyses using Tukey HSD pairwise comparisons for variable case studentized showed that the 
mean education was significantly lower in those with dementia at study entry $(\mathrm{M}=6.23, \mathrm{SD}=1.22)$ than in those who were free of dementia during the study period $(\mathrm{M}=7.28, \mathrm{SD}=2.46)$, but not significantly different from incident cases during the study period $(\mathrm{M}=6.91, \mathrm{SD}=1.81)$. Baseline age also differed significantly between groups.

\section{Cognitive trajectories on fluid and crystallized abilities in preclinical dementia}

Results from the multilevel analysis of the MMSE and fluid ability tests are displayed in Table 2.

A significant rate of cognitive decline was observed in all tests, crystallized or fluid, except Clock test, Figure Logic, Prose Recall and MIR Recognition. Figure 1 illustrates the expected trajectories of all neuropsychological tests investigated in these analyses from the time of study entry until the time of dementia diagnosis with the additional upper and lower bands of 5 years education.

Figure 1 about here

As illustrated in Figure 1 and listed in Table 2, mental status, measured with MMSE at the time of dementia diagnosis for a male participant age 83, with 7 years of education (i.e., reference values), enrolling in the study 5 years before dementia diagnosis was estimated at $23.04(\mathrm{SE}=0.90)$ with annual rate of decline of $-0.83(\mathrm{SE}=0.20)$ points.

The visuospatial ability and speed measured with Block Design and Digit Symbol at the time of diagnosis were estimated at $5.53(\mathrm{SE}=1.29)$ and $14.13(\mathrm{SE}=2.05)$ correspondingly, while the rates of decline were estimated at $-0.89(\mathrm{SE}=0.25)$ and $-0.84(\mathrm{SE}=0.44)$ points per year. $\mathrm{A}$ significant change was also observed in tests of memory recall and memory correspondence (measured with the Memory in Reality Tests) with the corresponding linear slope estimates of -0.55 $(\mathrm{SE}=0.13)$ and $-0.35(\mathrm{SE}=0.14)$ points per year, while the estimated mean intercepts were 2.43 $(\mathrm{SE}=0.58)$ and $4.14(\mathrm{SE}=0.56)$ respectively. 
Table 2 about here

Results from analysis of crystallized ability tests are displayed in Table 3. At dementia diagnosis, performance on Synonym test was estimated at 10.88 ( $\mathrm{SE}=0.90)$, while on the Information test was estimated at $20.33(\mathrm{SE}=1.97)$. The corresponding rates of decline were estimated at $-0.87(\mathrm{SE}=0.26)$ and $-1.78(\mathrm{SE}=0.31)$ points per year.

Table 3 about here

\section{Cognitive reserve: Education}

Education was positively associated with performance at time of dementia diagnosis on both crystallized measures (Synonym and Information Test, see Table 3). An increase in education from the average of 7 years (per extra year), was associated with a better performance on the Synonyms test with $0.80(\mathrm{SE}=0.35)$ and with $1.64(\mathrm{SE}=0.56)$ in the Information test.

In contrast, education did not have a significant association with the level of performance at the time of dementia diagnosis or with rate of change for any of the fluid or crystallized cognitive abilities examined, and this was not modified by excluding occupation from the existing models (see Tables 2 and 3).

\section{Cognitive reserve: Occupational class}

At the time of dementia diagnosis, individuals with higher occupational class (medium and high) had better performance on two measures of fluid abilities Figure Logic and Block Design (high occupational class only) and on one crystallized measure Synonym tests (medium occupation class only) compared to the estimated scores for individuals with lower occupational class, independent of level of education. Regarding the rate of preclinical decline, individuals with a 
medium occupational class declined at a slower annual rate on only two measures: Block Design and Synonym tests, compared to those with a lower occupational class.

\section{Other covariates}

Distance from baseline to the time of dementia diagnosis was associated only with rate of change on the Information test, with individuals declining at a faster rate with -0.22 ( $\mathrm{SE}=0.06)$ points per year closer to the time of diagnosis.

Women performed better than men at the time of dementia diagnosis, on memory correspondence $1.31(\mathrm{SE}=0.57)$, but worse on the Information test $-6.30(\mathrm{SE}=2.10)$, and did not change at a different rate compared to men with similar age, education and occupational levels.

Lastly, age at study entry was associated with performance at the time of dementia diagnosis for both MMSE and Block Design tests. Older age at study entry (per extra year increase from the age of 83 years) was associated with a lower performance on both MMSE and Block Design and a slightly faster decline in the Block Design test with $-0.15(\mathrm{SE}=0.06)$ points per year closer to dementia diagnosis.

\section{Discussion}

In this study, we examined the association between different markers of cognitive reserve, such as education and occupational class with preclinical cognitive decline in multiple tests of fluid and crystallized abilities, using a process-based approach where cognitive scores were modeled as a function of time to dementia diagnosis.

Firstly, we found no support for our hypothesis postulating that there will be a distinction between the rates of decline with fluid and crystallized cognitive abilities prior to the time of diagnosis, as seen in previous findings with normal aging (Horn \& Cattell, 1967). A significant rate of decline was observed for the MMSE and for both fluid and crystallized measures (Block Design, Digit Symbol, Memory Recall and Correspondence, Synonym and Information tests), even though the rates of decline varied by cognitive domain tapped by the various neuropsychological tests investigated. 
Secondly, our results consistently identified associations of education and performance among the crystallized abilities at the time of dementia diagnosis. However, education was not found to be associated with performance on fluid abilities at the time of dementia diagnosis nor with the rate of decline in either fluid or crystallized measures as we had originally hypothesized.

Thirdly, we found that occupational class did not mirror the effect of education in these analyses, suggesting a slightly more independent pattern from education itself. Interestingly, our results showed that higher levels of occupation (medium or high) were associated with better performance at the time of dementia diagnosis and with slower decline on both measures of fluid and crystallized abilities (e.g., Block Design and Synonym test). This could reflect the fact that there are separate effects for higher education and occupational attainment within the cognitive reserve hypothesis framework. According to cognitive reserve, the neuroprotective effect is assumed to reflect increased neural networks and hemispheric activation, in those with a higher number of years of education or higher occupation class, which tend to mask the dementia pathology leading perhaps to a slower rate of decline on both fluid and crystallized functions. Despite the fact that the majority of studies investigating the brain-reserve hypothesis have used education as a single measure, there is no overall consensus on how to measure cognitive reserve, and this remains a matter of ongoing debate. Only a limited number of studies have estimated cognitive reserve from multiple autobiographical data such as occupational complexity and frequency of mentally stimulating lifestyle pursuits along with education (Mortimer, 1997). As a consequence, cognitive reserve advantage cannot be conveyed to education alone, as this could continue to be affected by other circumstances such as socioeconomic circumstances unfolding across the lifespan. This highlights a number of methodological issues related to the components measured, when interpreting the role of cognitive reserve across different studies (Batterham, Mackinnon, \& Christensen, 2011; Carnero \& Del, 2007).

Furthermore, understanding the individual differences in cognitive decline prior to dementia diagnosis requires longer follow-up investigations, to be able to distinguish the severe 
neuropathological from the normal age-associated changes, which seem to be accelerated at least in some cases in later life (Hall et al., 2007; Schaie, Caskie, Revell, Willis, Kaszniak, \& Teri, 2005). In addition, it is essential to understand which cognitive abilities start to deteriorate faster with normal aging and which show further sensitivity in preclinical dementia. From a neurobiological perspective, cognitive decline is thought to reflect the accumulation of degeneration lesions, which could predict a faster process of mental deterioration associated with normal aging or neurodegeneration (Stern, Albert, Tang, \& Tsai, 1999; Wilson, Beck, Bienias, \& Bennett, 2007). However, only detailed longitudinal neuroimaging data could clarify the nature of neuropathological changes, when only relatively small impairments are detected in neuropsychological testing, particularly in individuals with high cognitive reserve that could have the potential to maintain peak cognitive functioning levels for an extended period of time, when confronted with brain neuropathology (Stern, 2012).

\section{Strengths and limitations}

In summary, the current findings provide rich and unique information regarding the rates of cognitive decline prior to dementia diagnosis and how we can detect evidence of variance across a wide range of cognitive abilities (both fluid and crystallized) within a sensitive time period in an

oldest-old population sample. The independent investigations of different markers of cognitive reserve (education and occupational class) on specific measures of cognitive abilities and their decline prior to dementia diagnosis have made salient contributions to the field of cognitive aging and dementia research.

A strength of this paper is the adoption of a process based approach to model cognitive decline documented as the optimal representation of intraindividual psychological change in individuals experiencing a common process that drives their cognitive changes, compared to the age-based models (Hall, Lipton, Sliwinski, \& Stewart, 2000; Sliwinski, Hofer, \& Hall, 2003). Using chronological age as the time metric in modeling cognitive change in older individuals at risk of dementia tends to obscure the true intra-individual trajectories and to produce misleading results, 
since participants of the same age are unlikely to be at the same stage in their disease progression. This improved methodology tends to overcome the large heterogeneity seen in observational studies, where cognition is modeled as a function of age (Sliwinski et al., 2003).

We are also aware of a number of limitations. The sample size available for these independent investigations was relatively small. However, the methodology employed compensated for the missing data aspect, since random effects mixed models handle naturally uneven spacing of repeated measurements, whether intentional or unintentional. The power to detect variance and covariance in rates of change was calculated for this study elsewhere and was favorable for the sample size used here (Rast \& Hofer, 2014).

Cognitive reserve was evaluated in this study with measures of education and occupational class. However, these two measures alone may not be sufficient to fully capture an individual's cognitive reserve accumulated across the life course. Furthermore, the results need to be considered within the historical context. This is a population sample born in 1913 and earlier in Sweden, where the attendance in school was compulsory only for children between 7-13 years of age, until 1960 (Erikson \& Jonsson, 1996). Therefore it can be considered a limitation of this study that the current sample had rather a reduced number of years of education and not a great variability. Lastly, we were not able to evaluate the cognitive changes within the monozygotic twins, since the initial low number of cases at the study entry and the various rates of drop out within pairs. This eliminated the possibility of examining the genetic effects and the genetic environment interaction within this population across the entire study period. However, a previous investigation comparing the OCTO-Twin Study with a population based sample of non-twins from the OCTO-Twin Study has indicated that the older surviving twins were similar to a representative sample of non-twins, based on health status, cognitive and behavioral functions (Simmons, Johansson, Zarit, Ljungquist, Plomin, \& McClearn, 1997), strengthening the case of generalizability of twin studies. 


\section{Conclusions}

We presented results from a longitudinal study with repeated measures of a wide range of fluid and crystallized abilities in the preclinical stages of dementia in which we investigated the role of different markers of cognitive reserve. We found that both fluid and crystallized cognitive abilities tend to decline in the period prior to dementia diagnosis. Importantly, education was positively associated with the level of performance on the crystallized measures even at the time of dementia diagnosis, but did not protect against faster rates of preclinical decline. However, occupational class did have a moderating role on the rate of decline in two of the fluid abilities measured. Thus, whereas both abilities decline during the preclinical phase, different markers of cognitive reserve have different moderating effects on the level of performance or on the rates of decline prior to dementia diagnosis. Longer follow-up periods and closer intervals between measurements are needed for more detailed characterizations of the transition from normal cognitive aging to the time at which there is evidence for a clinical dementia diagnosis. 


\section{References}

Albert, M. S., Jones, K., Savage, C. R., Berkman, L., Seeman, T., Blazer, D. et al. (1995). Predictors of cognitive change in older persons: MacArthur studies of successful aging. Psychol.Aging, 10, 578-589.

Anstey, K. J. \& Low, L. F. (2004). Normal cognitive changes in aging. Aust.Fam.Physician, $33,783-787$.

Batterham, P. J., Mackinnon, A. J., \& Christensen, H. (2011). The effect of education on the onset and rate of terminal decline. Psychol.Aging, 26, 339-350.

Bergem, A. L., Engedal, K., \& Kringlen, E. (1997). The role of heredity in late-onset Alzheimer disease and vascular dementia. A twin study. Arch.Gen Psychiatry, 54, 264-270.

Bjornsson, H. T., Fallin, M. D., \& Feinberg, A. P. (2004). An integrated epigenetic and genetic approach to common human disease. Trends Genet., 20, 350-358.

Carnero, P. C. \& Del, S. T. (2007). Education provides cognitive reserve in cognitive deterioration and dementia. Neurologia, 22, 78-85.

Cattell, R. B. (1963). Theory of fluid and crystallized intelligence: A critical experiment. Journal of Educational Psychology, 54, 1-22.

Clouston, S. A., Kuh, D., Herd, P., Elliott, J., Richards, M., \& Hofer, S. M. (2012). Benefits of educational attainment on adult fluid cognition: international evidence from three birth cohorts. Int.J Epidemiol., 41, 1729-1736.

Crawford, J. R., Deary, I. J., Starr, J., \& Whalley, L. J. (2001). The NART as an index of prior intellectual functioning: a retrospective validity study covering a 66-year interval. Psychol.Med., 31, 451-458.

Dureman, I. \& Salde, H. (1959). Psychometric and experimental methods for clinical use. Uppsala: Almqvist \& Wiskell. 
Erikson, R. \& Jonsson, J. O. (1996). The Swedish context: educational reform and long term change in educational inequality. In Can Education Be Equalized? The Swedish Case in Comparative Perspective ( Boulder: Westview Press).

Fleisher, A. S., Sowell, B. B., Taylor, C., Gamst, A. C., Petersen, R. C., \& Thal, L. J. (2007). Clinical predictors of progression to Alzheimer disease in amnestic mild cognitive impairment. Neurology, 68, 1588-1595.

Folstein, M. F., Folstein, S. E., \& McHugh, P. R. (1975). "Mini-mental state". A practical method for grading the cognitive state of patients for the clinician. J Psychiatr.Res., 12, 189-198.

Hall, C. B., Derby, C., LeValley, A., Katz, M. J., Verghese, J., \& Lipton, R. B. (2007). Education delays accelerated decline on a memory test in persons who develop dementia. Neurology, 69, 1657-1664.

Hall, C. B., Lipton, R. B., Sliwinski, M., \& Stewart, W. F. (2000). A change point model for estimating the onset of cognitive decline in preclinical Alzheimer's disease. Stat.Med, 19, 15551566.

Hofer, S. M. \& Piccinin, A. M. (2010). Toward an integrative science of life-span development and aging. J.Gerontol.B Psychol.Sci.Soc.Sci., 65B, 269-278.

Horn, J. L. \& Cattell, R. B. (1967). Age differences in fluid and crystallized intelligence. Acta Psychologica, 26, 107-129.

Johansson, B., Allen-Burge, R., \& Zarit, S. H. (1997). Self-reports on memory functioning in a longitudinal study of the oldest old: relation to current, prospective, and retrospective performance. J.Gerontol.B Psychol.Sci.Soc.Sci., 52, 139-146.

Johansson, B. \& Zarit, S. H. (1991). Dementia and cognitive impairment in the oldest old: a comparison of two rating methods. Int.Psychogeriatr., 3, 29-38.

Johansson, B. \& Zarit, S. H. (1995). Prevalence and incidence of dementia in the oldest old: A longitudinal study of a population-based sample of 84-90-year-olds in sweden. International Journal of Geriatric Psychiatry, 10, 359-366. 
Johansson, B., Zarit, S. H., \& Berg, S. (1992). Changes in cognitive functioning of the oldest old. Journal of Gerontology: Psychological Sciences, 75-80.

Jonsson, C. \& Molander, L. (1964). Manual till CVB-skalan Psykologi Forlaget (Manual of the CVB-Scales). Stockholm, Sweeden: Psykologi Forlaget.

McClearn, G. E., Johansson, B., Berg, S., Pedersen, N. L., Ahern, F., Petrill, S. A. et al. (1997). Substantial genetic influence on cognitive abilities in twins 80 or more years old. Science, 276, 1560-1563.

Mortimer, J. A. (1997). Brain reserve and the clinical expression of Alzheimer's disease. Geriatrics, 52 Suppl 2, S50-S53.

Pedersen, N. L., Lichtenstein, P., \& Svedberg, P. (2002). The Swedish Twin Registry in the third millennium. Twin.Res., 5, 427-432.

Petersen, R. C. (2004). Mild cognitive impairment as a diagnostic entity. Journal of Internal Medicine, 256, 183-194.

Piccinin, A. M., Muniz, G., Matthews, F. E., \& Johansson, B. (2011). Terminal decline from within- and between-person perspectives, accounting for incident dementia. J.Gerontol.B Psychol.Sci.Soc.Sci., 66, 391-401.

Rast, P. \& Hofer, S. M. (2014). Longitudinal design considerations to optimize power to detect variances and covariances among rates of change: simulation results based on actual longitudinal studies. Psychol.Methods, 19, 133-154.

Richards, M. \& Deary, I. J. (2005). A life course approach to cognitive reserve: A model for cognitive aging and development? Annals of Neurology, 58, 617-622.

Roberts, J. S., Karlawish, J. H., Uhlmann, W. R., Petersen, R. C., \& Green, R. C. (2010). Mild cognitive impairment in clinical care: a survey of American Academy of Neurology members. Neurology, 75, 425-431. 
Schaie, K. W., Caskie, G. I. L., Revell, A. U., Willis, S. L., Kaszniak, A. W., \& Teri, L. (2005). Extending neuropsychological assessments into the primary mental ability space. Aging Neuropsychology and Cognition, 12, 245-277.

Simmons, S. F., Johansson, B., Zarit, S. H., Ljungquist, B., Plomin, R., \& McClearn, G. E. (1997). Selection bias in samples of older twins? A comparison between octogenarian twins and singletons in Sweden. J Aging Health, 9, 553-567.

Sliwinski, M. J., Hofer, S. M., \& Hall, C. (2003). Correlated and coupled cognitive change in older adults with and without preclinical dementia. Psychol.Aging, 18, 672-683.

Sliwinski, M. J. \& Mogle, J. (2008). Time-based and process-based approaches to analysis of longitudinal data. In S.M.Hofer \& D. F. Alwin (Eds.), Handbook on cognitve aging: Interdisciplinary perspectives (pp. 477-491). Thousand Oaks: CA:Sage Publications.

Stern, Y. (2002). What is cognitive reserve? Theory and research application of the reserve concept. J Int Neuropsychol.Soc., 8, 448-460.

Stern, Y. (2009). Cognitive reserve. Neuropsychologia, 47, 2015-2028.

Stern, Y. (2012). Cognitive reserve in ageing and Alzheimer's disease. Lancet Neurol., 11, 1006-1012.

Stern, Y., Albert, S., Tang, M. X., \& Tsai, W. Y. (1999). Rate of memory decline in AD is related to education and occupation: cognitive reserve? Neurology, 53, 1942-1947.

Sweatt, J. D. (2010). Neuroscience. Epigenetics and cognitive aging. Science, 328, 701-702.

Tervo, S., Kivipelto, M., Hanninen, T., Vanhanen, M., Hallikainen, M., Mannermaa, A. et al. (2004). Incidence and risk factors for mild cognitive impairment: a population-based three-year follow-up study of cognitively healthy elderly subjects. Dement.Geriatr.Cogn Disord., 17, 196-203.

Valenzuela, M. J. \& Sachdev, P. (2006). Brain reserve and dementia: a systematic review. Psychol.Med., 36, 441-454.

van den Kommer, T. N., Bontempo, D. E., Comijs, H. C., Hofer, S. M., Dik, M. G., Piccinin, A. M. et al. (2009). Classification models for early identification of persons at risk for 
dementia in primary care: an evaluation in a sample aged 80 years and older. Dement.Geriatr.Cogn Disord., 28, 567.

Wechsler, S. (1981). WMS-R Wechsler adult intelligence scale-revised. San Antonio: The Psychological Corporation.

Wilson, R. S., Beck, T. L., Bienias, J. L., \& Bennett, D. A. (2007). Terminal cognitive decline: accelerated loss of cognition in the last years of life. Psychosom.Med., 69, 131-137.

Wilson, R. S., Li, Y., Aggarwal, N. T., Barnes, L. L., McCann, J. J., Gilley, D. W. et al. (2004). Education and the course of cognitive decline in Alzheimer disease. Neurology, 63, 11981202. 
Table 1

Characteristics of study participants

\begin{tabular}{|c|c|c|c|c|c|}
\hline \multirow[t]{4}{*}{ Total sample, N (\%) } & \multirow{4}{*}{$\begin{array}{l}\text { Total sample } \\
702(100 \%)\end{array}$} & \multirow{4}{*}{$\begin{array}{l}\text { Non-dementia } \\
477(68 \%)\end{array}$} & \multirow{2}{*}{\multicolumn{2}{|c|}{$\begin{array}{l}\text { Dementia cases } \\
225(32 \%)\end{array}$}} & \multirow[t]{4}{*}{$\mathrm{p}$ values } \\
\hline & & & & & \\
\hline & & & \multicolumn{2}{|c|}{ at study entry in study period } & \\
\hline & & & $\mathrm{N}=98$ & $\mathrm{~N}=127$ & \\
\hline Sex: Female, N & $468(66 \%)$ & $311(65 \%)$ & $78(76 \%)$ & $79(62 \%)$ & $\leq 0.01$ \\
\hline Baseline age, Mean (SD) & $83.52(3.2)$ & $83.42(3.2)$ & $84.64(3.4)$ & $83.13(2.6)$ & $\leq 0.001$ \\
\hline Education $^{1}$, Mean (SD) & $7.13(2.3)$ & $7.28(2.4)$ & $6.23(1.2)$ & $6.91(1.8)$ & $\leq 0.01$ \\
\hline Education, Range & 0 to $23 y$ & 0 to $23 y$ & 4 to $12 y$ & 2 to 17 & \\
\hline Occupation $^{2}$ Low, N (\%) & $332(49 \%)$ & $215(45 \%)$ & $35(58 \%)$ & $72(56 \%)$ & 0.09 \\
\hline Medium, N (\%) & $256(38 \%)$ & $197(41 \%)$ & $19(32 \%)$ & $40(32 \%)$ & \\
\hline High, N (\%) & $85(13 \%)$ & $64(14 \%)$ & $6(10 \%)$ & $15(12 \%)$ & \\
\hline Years to diagnosis from & - & - & - & -11 to $0.01 \mathrm{yrs}$ & \\
\hline study entry, Range/Mean & & & & -5 years & \\
\hline
\end{tabular}

Note. ${ }^{1}$ Education was coded as the number of years. ${ }^{2}$ Occupational class was coded into low (manual occupations), medium (non-manual occupations) and high (intermediate and professional occupations). 
Table 2

Mean, Standard Error of the Estimates of the Effect of Risk Factors on Random Effects of Preclinical Decline Mixed Model for the fluid cognitive abilities

\begin{tabular}{|c|c|c|c|c|c|c|c|c|c|}
\hline & \multicolumn{3}{|c|}{ MMSE } & \multicolumn{3}{|c|}{ Block Design } & \multicolumn{3}{|c|}{ Figure Logic } \\
\hline & \multicolumn{3}{|c|}{ Mental Status } & \multicolumn{3}{|c|}{ Visuo-spatial ability } & \multicolumn{3}{|c|}{ Inductive reasoning } \\
\hline & \multicolumn{3}{|c|}{$\mathrm{N}=126$} & \multicolumn{3}{|c|}{$\mathrm{N}=109$} & \multicolumn{3}{|c|}{$\mathrm{N}=91$} \\
\hline & Coef. & SE & $\mathrm{P}$ value & Coef. & SE & $P$ value & Coef. & SE & $\mathrm{P}$ value \\
\hline \multicolumn{10}{|l|}{ Fixed Effects } \\
\hline Level of performance at dementia & 23.04 & 0.90 & $<0.001$ & 5.53 & 1.29 & $<0.001$ & 14.14 & 0.99 & $<0.001$ \\
\hline \multicolumn{10}{|l|}{ diagnosis } \\
\hline Years to dementia diagnosis from & 0.00 & 0.17 & 0.99 & -0.36 & 0.24 & 0.13 & -0.21 & 0.19 & 0.26 \\
\hline \multicolumn{10}{|l|}{ study entry } \\
\hline Education & -0.00 & 0.25 & 0.99 & -0.29 & 0.39 & 0.46 & -0.50 & 0.34 & 0.14 \\
\hline Occupation Medium & 0.46 & 1.01 & 0.64 & 5.66 & 1.42 & $<0.001$ & 1.68 & 1.09 & 0.12 \\
\hline High & -1.41 & 1.48 & 0.34 & 3.76 & 2.21 & 0.08 & 3.96 & 2.04 & 0.05 \\
\hline Female & -0.47 & 0.95 & 0.61 & -1.58 & 1.34 & 0.24 & -0.32 & 1.05 & 0.75 \\
\hline Baseline age & -0.40 & 0.19 & 0.03 & -0.59 & 0.27 & 0.03 & 0.06 & 0.24 & 0.80 \\
\hline
\end{tabular}


COGNITIVE RESERVE AND COGNITIVE DECLINE

23

Rate of change

$-0.83 \quad 0.20$

$<0.001$

$\begin{array}{ll}-0.89 & 0.25\end{array}$

$<0.001$

$-0.23$

0.25

0.35

Years to diagnosis from study

$-0.04 \quad 0.03$

0.26

$-0.03$

0.05

0.57

$0.01 \quad 0.05$

0.85

entry

\begin{tabular}{|c|c|c|c|c|c|c|c|c|c|}
\hline Education & -0.04 & 0.04 & 0.35 & 0.02 & 0.07 & 0.75 & -0.04 & 0.09 & 0.65 \\
\hline Occupation Medium & -0.06 & 0.20 & 0.76 & 0.55 & 0.24 & 0.02 & 0.29 & 0.25 & 0.24 \\
\hline High & -0.32 & 0.32 & 0.31 & 0.51 & 0.47 & 0.27 & 0.13 & 0.48 & 0.77 \\
\hline Female & -0.03 & 0.04 & 0.44 & -0.16 & 0.24 & 0.49 & 0.14 & 0.24 & 0.55 \\
\hline Baseline age & -0.03 & 0.04 & 0.44 & -0.15 & 0.06 & 0.01 & 0.03 & 0.07 & 0.63 \\
\hline Random Effects Variance & - & - & $95 \% \mathrm{CI}$ & - & - & $95 \% \mathrm{CI}$ & - & - & $95 \% \mathrm{CI}$ \\
\hline Level of performance & 0.56 & 0.09 & $0.40-0.79$ & 0.04 & 0.02 & $0.24-0.52$ & 0.31 & 0.12 & $0.15-0.68$ \\
\hline Rate of decline & 3.84 & 0.40 & $3.12-4.72$ & 5.15 & 0.43 & $4.37-6.07$ & 2.73 & 0.40 & $2.04-3.63$ \\
\hline Error & 2.82 & 0.15 & $2.54-3.13$ & 3.20 & 0.19 & $2.84-3.61$ & 3.20 & 0.22 & $2.79-3.67$ \\
\hline
\end{tabular}


Table 2 (continued)

Mean, Standard Error of the Estimates of the Effect of Risk Factors on Random Effects of Preclinical Decline Mixed Model for the fluid cognitive abilities

\begin{tabular}{|c|c|c|c|c|c|c|c|c|c|}
\hline & \multicolumn{3}{|c|}{ Clock } & \multicolumn{3}{|c|}{ Digit Symbol } & \multicolumn{3}{|c|}{ Prose Recall } \\
\hline & \multicolumn{3}{|c|}{ Visuo-constructive ability } & \multicolumn{3}{|c|}{ Short-term memory } & \multicolumn{3}{|c|}{ Verbal memory } \\
\hline & \multicolumn{3}{|c|}{$\mathrm{N}=120$} & \multicolumn{3}{|c|}{$\mathrm{N}=110$} & \multicolumn{3}{|c|}{$\mathrm{N}=110$} \\
\hline & Coef. & $\mathrm{SE}$ & $\mathrm{P}$ value & Coef. & SE & $P$ value & Coef. & $\mathrm{SE}$ & $\mathrm{P}$ value \\
\hline \multicolumn{10}{|l|}{ Fixed Effects } \\
\hline Level of performance at dementia & 11.98 & 0.71 & $<0.001$ & 14.13 & 2.05 & $<0.001$ & 5.67 & 1.01 & $<0.001$ \\
\hline \multicolumn{10}{|l|}{ diagnosis } \\
\hline Years to dementia diagnosis from & -0.04 & 0.13 & 0.75 & -0.15 & 0.37 & 0.67 & -0.06 & 0.18 & 0.72 \\
\hline \multicolumn{10}{|l|}{ study entry } \\
\hline Education & -0.22 & 0.25 & 0.37 & 1.30 & 0.82 & 0.11 & 0.19 & 0.32 & 0.55 \\
\hline Occupation Medium & 0.45 & 0.81 & 0.58 & 4.01 & 2.30 & 0.08 & -0.29 & 1.06 & 0.78 \\
\hline High & -0.45 & 1.19 & 0.70 & -0.04 & 3.31 & 0.98 & 0.47 & 1.62 & 0.76 \\
\hline Female & -0.53 & 0.73 & 0.46 & -0.98 & 2.05 & 0.63 & 0.14 & 1.02 & 0.89 \\
\hline Baseline age & -0.03 & 0.15 & 0.81 & -0.43 & 0.47 & 0.36 & -0.24 & 0.20 & 0.23 \\
\hline
\end{tabular}




\begin{tabular}{|c|c|c|c|c|c|c|c|c|c|}
\hline Rate of change & -0.26 & 0.17 & 0.12 & -0.84 & 0.44 & 0.05 & -0.44 & 0.25 & 0.07 \\
\hline Years to diagnosis from study & -0.02 & 0.03 & 0.52 & -0.02 & 0.08 & 0.75 & -0.01 & 0.04 & 0.71 \\
\hline \multicolumn{10}{|l|}{ entry } \\
\hline Education & -0.00 & 0.04 & 0.96 & 0.05 & 0.13 & 0.65 & -0.00 & 0.06 & 0.96 \\
\hline Occupation Medium & 0.10 & 0.17 & 0.56 & -0.32 & 0.39 & 0.42 & -0.17 & 0.24 & 0.48 \\
\hline High & -0.38 & 0.28 & 0.18 & -0.87 & 0.69 & 0.21 & -0.09 & 0.38 & 0.80 \\
\hline Female & -0.10 & 0.16 & 0.54 & -0.30 & 0.40 & 0.45 & -0.19 & 0.24 & 0.42 \\
\hline Baseline age & 0.02 & 0.04 & 0.53 & 0.09 & 0.10 & 0.37 & -0.01 & 0.05 & 0.79 \\
\hline Random Effects Variance & - & - & $95 \% \mathrm{CI}$ & - & - & $95 \% \mathrm{CI}$ & - & - & $95 \% \mathrm{CI}$ \\
\hline Level of performance & 0.40 & 0.08 & $0.27-0.59$ & 0.17 & 0.21 & $0.01-1.90$ & 0.56 & 0.16 & $0.32-0.99$ \\
\hline Rate of decline & 2.48 & 0.27 & $1.99-3.04$ & 7.25 & 0.84 & $5.77-9.11$ & 3.37 & 0.50 & $2.52-4.52$ \\
\hline Error & 2.50 & 0.14 & $2.23-2.80$ & 4.89 & 0.32 & $4.30-5.56$ & 2.59 & 0.20 & $2.22-3.02$ \\
\hline
\end{tabular}


Table 2 (continued)

Mean, Standard Error of the Estimates of the Effect of Risk Factors on Random Effects of Preclinical Decline Mixed Model for the fluid cognitive abilities

\begin{tabular}{|c|c|c|c|c|c|c|c|c|c|}
\hline & \multicolumn{3}{|c|}{ Memory Recall } & \multicolumn{3}{|c|}{ Memory Recognition } & \multicolumn{3}{|c|}{ Memory Correspondence } \\
\hline & \multicolumn{3}{|c|}{ Short-term memory } & \multicolumn{3}{|c|}{ Short-term memory } & \multicolumn{3}{|c|}{ Short-term memory } \\
\hline & \multicolumn{3}{|c|}{$\mathrm{N}=112$} & \multicolumn{3}{|c|}{$\mathrm{N}=113$} & \multicolumn{3}{|c|}{$\mathrm{N}=110$} \\
\hline & Coef. & $\mathrm{SE}$ & $\mathrm{P}$ value & Coef. & SE & $P$ value & Coef. & SE & $P$ value \\
\hline \multicolumn{10}{|l|}{ Fixed Effects } \\
\hline Level of performance at dementia & 2.43 & 0.58 & $<0.001$ & 8.60 & 0.43 & $<0.001$ & 4.14 & 0.56 & $<0.001$ \\
\hline \multicolumn{10}{|l|}{ diagnosis } \\
\hline Years to dementia diagnosis from & -0.00 & 0.11 & 0.95 & -0.13 & 0.08 & 0.09 & 0.09 & 0.10 & 0.35 \\
\hline \multicolumn{10}{|l|}{ study entry } \\
\hline Education & 0.21 & 0.21 & 0.31 & 0.20 & 0.15 & 0.19 & 0.29 & 0.21 & 0.16 \\
\hline Occupation Medium & 0.52 & 0.65 & 0.42 & -0.69 & 0.47 & 0.14 & 0.14 & 0.62 & 0.81 \\
\hline High & -0.71 & 0.99 & 0.47 & -0.01 & 0.75 & 0.98 & -0.32 & 0.94 & 0.73 \\
\hline Female & 0.41 & 0.60 & 0.49 & -0.19 & 0.44 & 0.66 & 1.31 & 0.57 & 0.02 \\
\hline Baseline age & -0.09 & 0.12 & 0.43 & -0.17 & 0.09 & 0.07 & -0.10 & 0.12 & 0.38 \\
\hline
\end{tabular}




\begin{tabular}{|c|c|c|c|c|c|c|c|c|c|}
\hline Rate of change & -0.55 & 0.13 & $<0.001$ & -0.13 & 0.10 & 0.21 & -0.35 & 0.14 & 0.01 \\
\hline Years to diagnosis from study & -0.04 & 0.02 & 0.10 & -0.02 & 0.02 & 0.34 & -0.02 & 0.03 & 0.48 \\
\hline \multicolumn{10}{|l|}{ entry } \\
\hline Education & 0.01 & 0.03 & 0.65 & 0.03 & 0.02 & 0.27 & 0.02 & 0.04 & 0.57 \\
\hline Occupation Medium & 0.09 & 0.14 & 0.48 & -0.06 & 0.10 & 0.56 & -0.02 & 0.15 & 0.85 \\
\hline High & 0.02 & 0.23 & 0.89 & 0.01 & 0.18 & 0.92 & -0.20 & 0.24 & 0.41 \\
\hline Female & -0.07 & 0.13 & 0.60 & -0.07 & 0.10 & 0.50 & -0.03 & 0.14 & 0.83 \\
\hline Baseline age & 0.05 & 0.03 & 0.06 & -0.02 & 0.02 & 0.23 & 0.02 & 0.03 & 0.42 \\
\hline Random Effects Variance & - & - & $95 \% \mathrm{CI}$ & - & - & $95 \% \mathrm{CI}$ & - & - & $95 \% \mathrm{CI}$ \\
\hline Level of performance & 0.30 & 0.07 & $0.17-0.50$ & 0.33 & 0.05 & $0.23-0.46$ & 0.27 & 0.10 & $0.12-0.59$ \\
\hline Rate of decline & 2.29 & 0.27 & $1.80-2.90$ & 1.32 & 0.20 & $0.98-1.77$ & 1.78 & 0.29 & $1.29-2.47$ \\
\hline Error & 1.54 & 0.10 & $1.34-1.77$ & & & $1.32-1.71$ & 1.72 & 0.12 & $1.48-1.99$ \\
\hline
\end{tabular}


Table 3

Mean, Standard Error of the Estimates of the Effect of Risk Factors on Random Effects of Preclinical Decline Mixed Model for the crystallized cognitive abilities

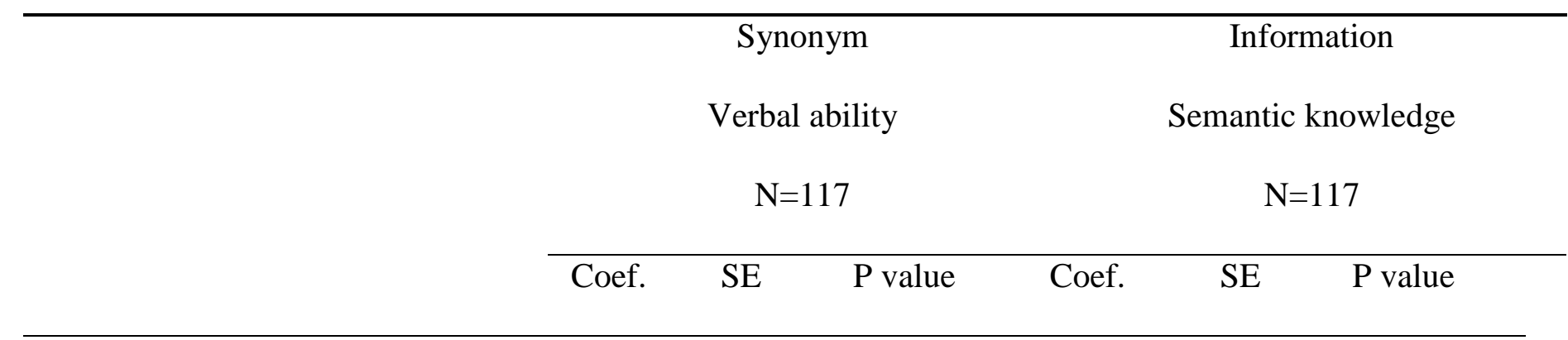

Fixed Effects

Level of performance at dementia

$10.88 \quad 1.15$

$<0.001$

20.33

1.97

$<0.001$

diagnosis

Years to dementia diagnosis from

$-0.10 \quad 0.22$

0.63

$\begin{array}{ll}-0.22 & 0.39\end{array}$

0.56

study entry

\begin{tabular}{lcccccc} 
Education & 0.80 & 0.35 & 0.02 & 1.64 & 0.56 & 0.004 \\
Occupation Medium & 3.12 & 1.28 & 0.01 & 3.57 & 2.25 & 0.11 \\
\multicolumn{1}{c}{ High } & 3.09 & 2.17 & 0.15 & 1.95 & 3.24 & 0.55 \\
Female & 1.36 & 1.24 & 0.27 & -6.30 & 2.10 & 0.003
\end{tabular}




$\begin{array}{lllllll}\text { Baseline age } & 0.19 & 0.26 & 0.46 & -0.75 & 0.43 & 0.08 \\ \text { Rate of change } & -0.87 & 0.26 & <0.001 & -1.78 & 0.31 & <0.001 \\ \text { Years to diagnosis from study } & -0.07 & 0.05 & 0.18 & -0.22 & 0.06 & 0.001 \\ \text { entry } & & & & & & \\ \text { Education } & -0.07 & 0.07 & 0.32 & -0.09 & 0.08 & 0.27 \\ \text { Occupation Medium } & 0.63 & 0.24 & 0.01 & -0.03 & 0.30 & 0.91 \\ \quad 0.64 & 0.47 & 0.18 & -0.62 & 0.50 & 0.21 \\ \quad \text { High } & 0.27 & 0.25 & 0.27 & 0.27 & 0.29 & 0.35 \\ \text { Female } & 0.06 & 0.06 & 0.35 & -0.02 & 0.07 & 0.76 \\ \text { Baseline age } & - & - & 95 \% \mathrm{CI} & - & - & 95 \% \mathrm{CI} \\ \text { Random Effects Variance } & 0.34 & 0.19 & 0.11-1.06 & 0.23 & 0.49 & 0.00-15.05 \\ \text { Level of performance } & 4.40 & 0.51 & 3.50-5.53 & 9.52 & 0.77 & 8.11-11.18 \\ \text { Rate of decline } & 2.36 & 0.21 & 1.98-2.82 & 3.81 & 0.28 & 3.29-4.42 \\ \text { Error } & & & & & & \end{array}$


Figure 1. Estimated mean trajectories of cognitive decline prior to dementia diagnosis for a man aged 83 with 7 years of education entering the study at 5 years from dementia diagnosis (solid lines) and individuals with 2 and 12 years of education (the additional dotted upper and lower lines).

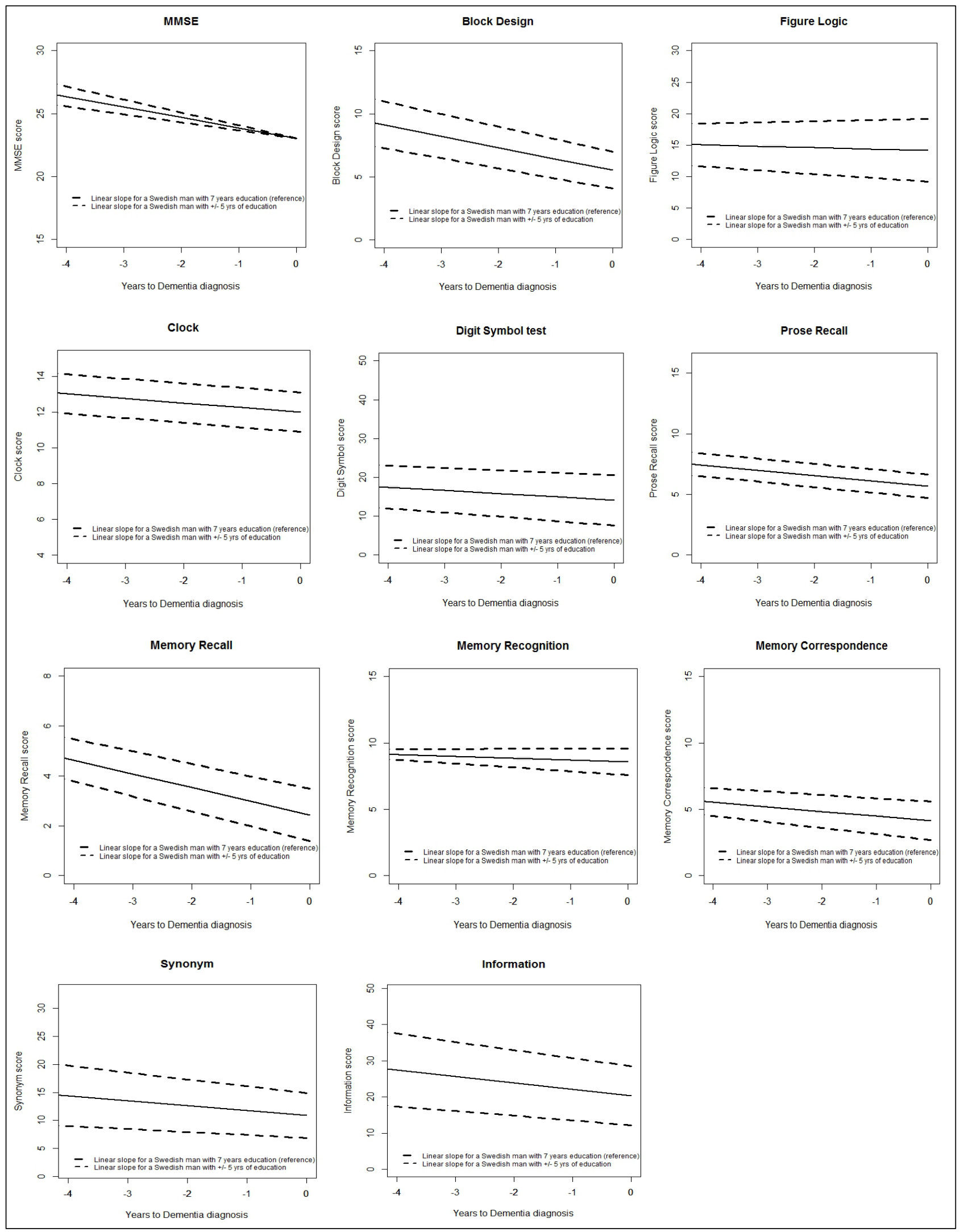

